\title{
The role of hepatic trans-arterial chemoembolization in metastatic medullary thyroid carcinoma: a specialist center experience and review of the literature
}

\author{
S Grozinsky-Glasberg1, * A I Bloom², *, N Lev-Cohain², A Klimov², H Besiso' \\ and D J Gross ${ }^{1}$ \\ ${ }^{1}$ Neuroendocrine Tumor Unit, Endocrinology \& Metabolism Service, Department of Medicine, and \\ ${ }^{2}$ Department of Radiology, Hadassah-Hebrew University Medical Center, Jerusalem, Israel \\ *(S Grozinsky-Glasberg and A I Bloom contributed equally to this work)
}

\author{
Correspondence \\ should be addressed \\ to S Grozinsky-Glasberg \\ Email \\ simonag@hadassah.org.il
}

\section{Abstract}

Background: Liver metastases are relatively common in patients with metastatic medullary thyroid carcinoma (MTC), carrying a negative impact on disease prognosis. The options for selective therapy of liver metastases in MTC patients are limited to catheter-guided procedures such as trans-arterial chemoembolization (TACE). Data regarding the effectiveness and safety of this procedure in MTC are limited.

Aim: To explore the clinical outcome, survival and safety profile of TACE for liver metastases in a group of MTC patients.

Methods: Retrospective case series of patients treated at a single tertiary University Medical Center from 2005 to 2015. Results: Seven consecutive patients (mean age $64.5 \pm 10.9$ years, 5 females) with histologically confirmed MTC with liver metastases were included. Metastatic involvement of the liver was less than $50 \%$ of the liver volume in all patients. The median size of the largest liver lesion was $40 \pm 6.9 \mathrm{~mm}$. The patients underwent in total 20 sessions of TACE. Clinical improvement as well as tumor response (PR) were observed in all patients. The median time to tumor progression was 38 months (range 8-126). Three patients were still alive at the end of the follow-up period (a median overall survival rate of $57 \pm 44$ months).

Conclusion: TACE in MTC patients with hepatic metastases is usually well tolerated and induces both clinical improvement and tumor response for prolonged periods of time in the majority of patients. This therapeutic option should always be considered, irrespective of the presence of extrahepatic metastasis.

\section{Introduction}

Medullary thyroid carcinoma (MTC) is a rare calcitoninsecreting neuroendocrine tumor derived from the parafollicular $\mathrm{C}$ cells of the thyroid gland. Although MTC accounts for only 3-10\% of all thyroid carcinomas, it is responsible for up to $13.4 \%$ of all thyroid cancerrelated deaths $(1,2,3,4)$. The majority of patients $(85 \%)$ have sporadic MTC, whereas hereditary MTC is usually
() 2017 European Society of Endocrinology Printed in Great Britain diagnosed earlier as a component of the type 2 multiple endocrine neoplasia (MEN) syndromes, MEN2A, MEN2B and the related familial MTC (FMTC) syndrome (5). The clinical course varies according to certain patient- and disease-related factors, such as age at diagnosis, disease extension and extent of surgery, though these are still controversial (2). Distant metastases (to the lungs, bones 
and liver) are present in up to $15 \%$ at disease presentation in patients with MTC, being associated with persistent loco-regional disease $(6,7,8)$. Patients with distant metastases at diagnosis have a poor prognosis, with only $20-40 \%$ surviving at 10 years $(9,10)$.

The $\mathrm{C}$ cells of the thyroid may secrete a variety of hormones or biogenic amines, mainly calcitonin (CLT) and carcinoembryonic antigen (CEA), which are valuable tumor markers, and rarely adrenocorticotropic hormone (ACTH), B-melanocyte-stimulating hormone, chromogranin, histaminase, neurotensin and somatostatin $(11,12,13)$.

The primary treatment for MTC is extensive and meticulous surgical resection $(14,15,16)$. However, patients with progressive or symptomatic metastatic disease who cannot be treated by surgery are usually considered as candidates for systemic therapy; there is a limited role for external-beam radiotherapy in patients with locally aggressive disease $(17,18)$.

In patients with symptomatic and progressive metastatic disease, treatment with tyrosine kinases inhibitors (TKIs) targeting both RET and VEGFR should be considered as systemic therapy. The TKIs vandetanib or cabozantinib can be used as single agent first-line systemic therapy in patients with advanced progressive MTC $(19,20,21)$. Single agent or combination cytotoxic chemotherapeutic regimens (e.g. combination therapy with doxorubicin, 5FU and dacarbazine, streptozotocin, cyclophosphamide or vincristine) are characterized by low- and short-duration response rates (15-20\%), although they may be indicated in selected patients $(22$, $23,24)$. Several studies have demonstrated that thyroid tumor cells may express somatostatin receptors; therefore, the utilization of peptide receptor radionuclide therapy (PRRT) with different types of radiolabeled somatostatin analogues may be suggested in selective patients with adequate SSTR expression (25). Noteworthy, although over $90 \%$ of MTCs express cholecystokinin (CCK)-B/gastrin receptors, there is still very limited data on the potential benefit of radiolabeled minigastrin administration in patients with metastatic progressive MTC (26).

Liver metastases occur in $\sim 45 \%$ of patients with advanced MTC, are often multiple and disseminated throughout the parenchyma and are usually not amenable to surgery or radiofrequency ablation (27). When progressive liver disease occurs (with increase in the size of the metastases, with/without symptoms such as diarrhea, flushing or abdominal pain), treatment with either locoregional selective arterial chemo-/embolization (TACE or TAE respectively), selective internal radiation therapy
(SIRT) or systemic therapy should be considered $(28,29)$. The possible relative benefit of either of these treatments (TACE/TAE or SIRT) needs to be explored.

Data regarding the effectiveness and safety of trans-arterial loco-regional therapy in patients with advanced unresectable MTC are limited. We conducted a retrospective analysis to explore the clinical outcome, survival and safety profile of TACE for liver metastases in a group of consecutive patients with metastatic MTC. We have also reviewed the literature to compare our cohort of patients' characteristics with those previously reported.

\section{Subjects and methods}

Seven consecutive patients with MTC diagnosed and treated at a tertiary referral center in Israel have been retrospectively studied for a period of 10 years (between 2005 and 2015). Clinical characteristics of the patients, biochemical and radiological assessment, as well as histopathological findings were all analyzed. The application of therapeutic modalities (e.g. surgery, irradiation therapy, somatostatin receptor analogues, chemotherapy, trans-arterial chemoembolization, etc.), as well as the long-term outcome, were determined.

This study was approved by the IRB according to WMA Helsinki guidelines for research in human subjects.

\section{Clinical assessment}

To define symptomatic or asymptomatic patients with MTC, patients' files were reviewed for the presence of symptoms compatible with metastatic MTC at diagnosis, including dyspnea, cough, diarrhea, flushing or bone pain. In the absence of typical symptoms, the reason for performing an imaging test was recorded. The presence of other endocrine disorders or malignancies in the patients and their families were also recorded.

\section{Biochemical evaluation}

All patients underwent a complete biochemical assessment. Serum calcitonin (CLT) levels were measured before treatment with TACE after an overnight fast, and thereafter at regular intervals (3-6 months) during the follow-up period. Serum CLT was measured using a commercially available radioimmunoassay ELSA-CT kit (Cis-Bio International, Gifsur-Yvette, France; normal $0-1.46 \mathrm{pmol} / \mathrm{L}$ ). CEA was collected in all patients, at diagnosis and also periodically at 3-6 months during the 
study period (measured using the Enzymum-test CEA kit (Boehringer, Mannheim, Germany; normal 0-3 ng/mL)).

Liver enzymes (alkaline phosphatase, GGT, GOT and GPT) have also been evaluated before and after each treatment, and then after 2-4 weeks and regularly at a 3to 6-month interval after each treatment. All patients had reasonable liver function tests before TACE, up to X1.5 of the upper normal range.

\section{Radiological assessment}

All patients underwent a high-resolution cross-sectional computerized tomography (CT) and/or a magnetic resonance imaging (MRI) of the liver, as well as a Doppler ultrasonography of the liver before the initiation of therapy, to assess the size, the extent as well as the accurate segmental localization of the tumor and to evaluate the macroscopic vascular invasion into the hepatic and portal veins. The number of nodules, size, location, edge, the presence of calcifications, as well as the patency of the liver vasculature have been recorded. Some of the patients also underwent a functional imaging $\left({ }^{68} \mathrm{Ga}\right.$-DOTATATEPET-CT, ${ }^{18}$ F-DOPA-PET-CT, or FDG-PET-CT) (Fig. 1).

\section{TACE procedure}

\section{Indications}

Patients with preserved liver function in the presence of hyper-vascular progressive and/or symptomatic liver metastases, where the intrahepatic component was a main source of morbidity and mortality, were included and underwent TACE (30).

\section{Contraindications}

Patients with a massive or diffuse metastatic involvement of more than $50 \%$ of liver, with suspicion of impending hepatic insufficiency/failure, portal vein invasion, high serum bilirubin (more than $3-5 \mathrm{mg} / \mathrm{dL}$ ), high levels of serum LDH (more than $425 \mathrm{IU} / \mathrm{L}$ ) and transaminases (more than $100 \mathrm{IU} / \mathrm{L}$ ), as well as known contraindications to the chemotherapeutic agent or known allergic reactions to the contrast media were excluded (31).

\section{Chemoembolization method}

Hepatic arterial TACE was performed through a femoral access using a 5 French catheter. A portogram was first obtained by superior mesenteric artery injection of contrast medium to confirm the patency of the portal trunk and its branches, and an angiogram of the hepatic artery was obtained to serve as a map to guide the TACE; if a prominent arterio-portal shunt was observed, it was selectively embolized prior to treatment. Usually, as tumor liver involvement was over $30 \%$, selective TACE of one liver lobe at a time was performed. For each single course of TACE, the cytotoxic agent (mitomycin $30 \mathrm{mg}$ / treatment or doxorubicin $50 \mathrm{mg} /$ treatment) was mixed with 7-10 mL of iodized oil (Lipiodol; Guerbet, Aulnay sous Bois, France) to obtain a water-in-oil emulsion; after injection of the emulsion, embolization was performed using gel foam powder to complete blood flow stagnation.

The contralateral liver lobe was usually treated 1-3 months later, according to the tolerance of the first TACE session.

\section{Peri-procedure medical protocol}

Pre- and post-procedure care included fluid infusion, antiemetics, pain control including narcotic analgesics, as well as oral dexamethasone (at a dosage of $4 \mathrm{mg}, 2-3$ time/ day) aimed to alleviating the post-embolization syndrome (PES) (nausea, vomiting, fever, abdominal pain and anorexia) and to improve liver function after TACE (32). Patients also received prophylactic antibiotic infusion the morning of the procedure, to prevent liver abscess, cholangitis or frank sepsis (33).

\section{Evaluation of response to TACE}

In all cases, the response to treatment was assessed based on contrast enhancement CT and/or on MRI performed at 2-3 months and then every 6 months and was defined using established WHO criteria, as follows: (1) complete response (CR) (complete regression of all clinical, radiological and hormonal evidence of the tumor); (2) partial response (PR) (a 50\% or greater reduction in all measurable tumor, clinical symptoms and hormonal levels, with no appearance of new lesions); (3) stable disease (SD) (less than 50\% reduction or no greater than $25 \%$ increase in tumor size, clinical symptoms and hormonal measurements) and (4) tumor progression (progressive disease, PD) (appearance of new lesions or an increase of $25 \%$ or more in tumor size and clinical/ hormonal deterioration) (34).

In the case of tumor response (CR, PR or SD), TACE was not performed again until disease progression occurred, at which time, a new cycle of TACE was considered; in the case of PD, treatment was discontinued. 

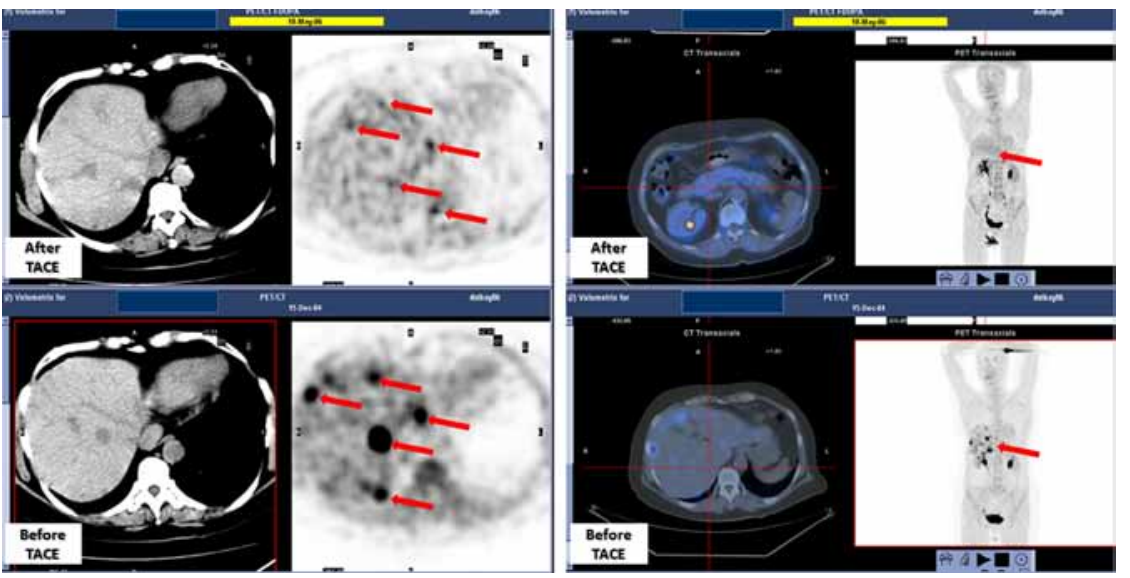

Figure 1

The F-DOPA PET/CT images of one of our patients, before and 1.5 years after TACE, demonstrating significant response with reduction in the number and size of liver metastases. A full colour version of this figure is available at http://dx.doi. org/10.1530/EJE-16-0960.
Clinical response was defined as a decrease by $\geq 25 \%$ in the number of stools or flushing attacks per day. Biological response was defined as a decrease of $\geq 25 \%$ in the CLT and CEA levels.

\section{Statistical analysis}

Results were expressed as mean \pm s.D. Differences in variables were analyzed by the chi-square test and Student's $t$-test using the SigmaStat 2.03 computerized program (Systat Software Inc., Point Richmond, CA, USA). A $P$ value of $<0.05$ was considered significant.

\section{Results}

The clinical characteristics of the patients with liver metastatic MTC are shown in Table 1. Five out of the seven patients were female, with a mean age of $64.5 \pm 10.9$ years (range 52-78). Six patients had sporadic MTC based on negative screening for germline RET mutation, whereas one patient had multiple endocrine neoplasia MEN2A with a RET mutation at the 634 codon, and who

Table 1 The clinical characteristics of the patients $(n=7)$ with liver metastatic MTC included in the study. Data are presented as mean \pm s.D. or as $n(\%)$

\begin{tabular}{l}
\hline Characteristics \\
\hline Age (years) \\
Female \\
Caucasians \\
Size of largest liver lesion (range) \\
Duration of liver involvement before \\
first TACE (months) \\
Previous thyroidectomy and neck \\
dissection \\
Other metastatic sites, bones \\
Symptomatic \\
Familial aggregation
\end{tabular}

\begin{tabular}{c}
\hline Values \\
\hline $64.5 \pm 10.9$ \\
$5(71 \%)$ \\
$7(100 \%)$ \\
$29.5 \pm 18 \mathrm{~mm}(13-60)$ \\
$30 \pm 24.5$ \\
$7(100 \%)$ \\
$4 / 7(57 \%)$ \\
$5(71 \%)$ \\
$1(14 \%)$ \\
\hline
\end{tabular}

underwent previous bilateral adrenalectomy $\mathrm{d} / \mathrm{t}$ bilateral pheochromocytomas. All the included patients previously underwent total thyroidectomy and neck lymph nodes dissection.

Hepatic single metastasectomy was performed previously in one of the included patients (patient 1), as in most patients, the liver metastases were multiple and diffuse in both lobes. One patient (patient 2) has been treated with systemic chemotherapy with adriamycin, without any objective response; the length of time between the termination of chemotherapy and TACE was 26 months. None of the included patients received tyrosine kinase inhibitors before TACE.

Metastatic involvement of the liver determined on CT or MRI was less than $50 \%$ of the liver volume, and bilateral (involving both hepatic lobes), in all patients. The size of the largest liver lesion ranged from 30 to $45 \mathrm{~mm}$ (median, $40 \pm 6.9 \mathrm{~mm}$ ). All patients had appropriate liver function tests ( $\leq 1.5$ upper normal values).

All patients had radiological and biochemical progression (PD) at the time of the first TACE (WHO criteria). The mean duration of liver metastases before the first TACE was 30 months (range 4-72), whereas the time interval between the MTC diagnosis and first TACE was 63 months (range 24-132).

Four out of the seven patients had also bone involvement. Five patients were symptomatic before the first TACE, in terms of weights loss, weakness, abdominal pain, diarrhea or flushing, and elevation in the levels of CLT and CEA have been observed in all included patients.

\section{Chemoembolization}

A total of 20 courses of TACE (mean, 2.8; range, $2-5$ per patient) were performed and were delivered during 11 cycles during the study period. They were technically 


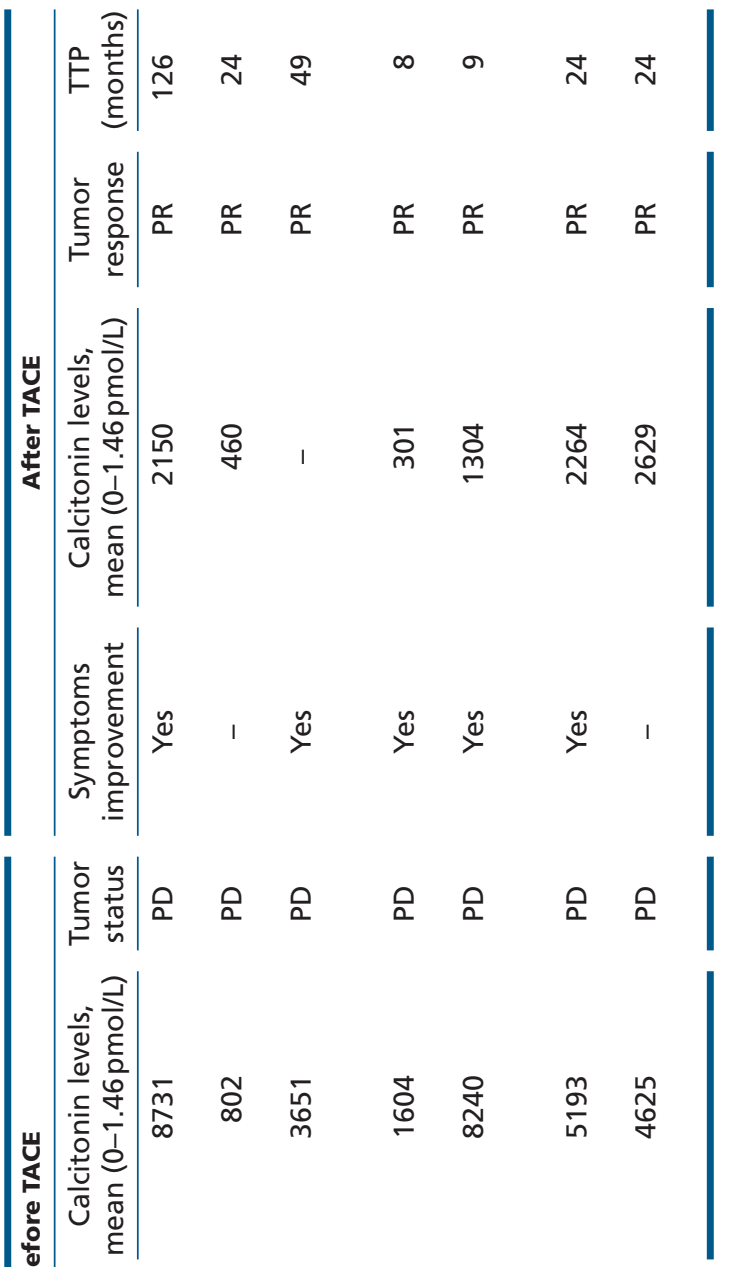

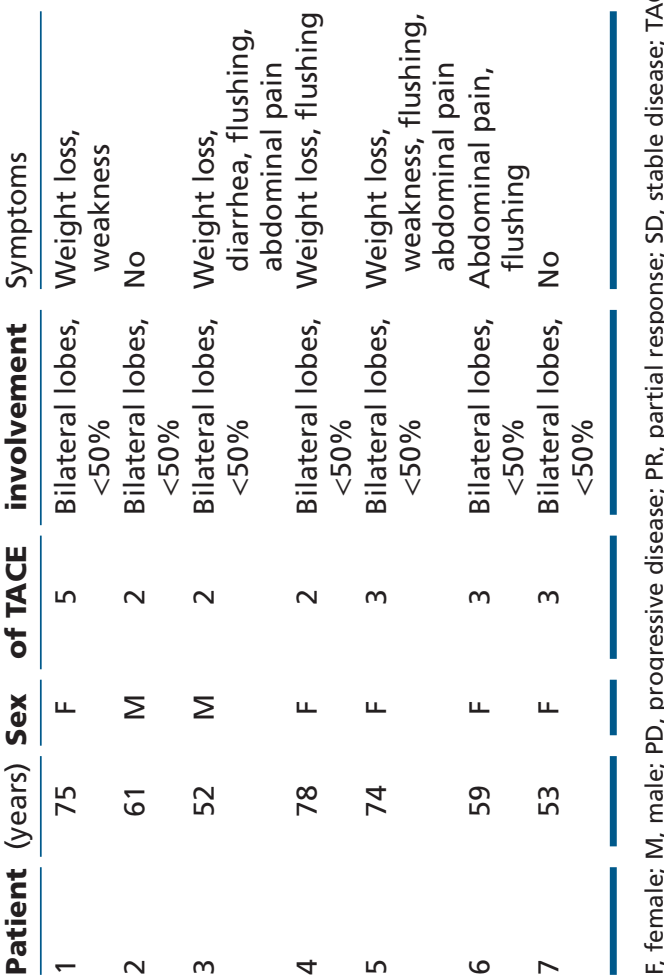

successful in all cases. In one patient, TACE was performed using $15 \mathrm{mg}$ of mitomycin/lipiodol emulsion injection followed by gel foam, whereas in the other six patients, TACE was performed using the injection of $50 \mathrm{mg}$ of adriamycin/lipiodol emulsion, followed by gel foam. (Table 2)

After embolization, all patients experienced PES with symptoms (nausea, vomiting, abdominal pain and fever) that were usually mild to moderate (grade 1-2) and usually transient, in parallel with the elevation of liver enzymes that normalized/decreased within 7-30days. One patient (case 1) developed an increase in the blood pressure up to $190 / 110 \mathrm{mmHg}$ that resolved with treatment with Pressolat (nifedipine). Hospitalization lasted between 3 and 5 days after embolization.

\section{Response to TACE}

\section{Clinical response}

Clinical response was observed in all symptomatic patients, including improvement in the general condition, and specifically in symptoms of weakness, abdominal pain, tiredness, flushing or diarrhea.

\section{Tumor markers}

Before the first TACE, CLT levels were elevated in all patients reaching levels of $4615 \pm 3122 \mathrm{pmol} / \mathrm{L}$ (mean \pm s.D., normal range: $0-1.46 \mathrm{pmol} / \mathrm{L}$ ), whereas at last follow-up, CLT levels significantly decreased and reached $1803 \pm 1142 \mathrm{pmol} / \mathrm{L}$. Plasma CEA levels before TACE were also increased up to $787 \pm 702 \mathrm{ng} / \mathrm{mL}$ (normal range: $0-3 \mathrm{ng} / \mathrm{mL}$ ) and decreased after TACE up to $372 \pm 543 \mathrm{ng} / \mathrm{mL}$.

\section{Disease status during the follow-up period}

At the end of the all treatments, partial tumor response was observed in all patients, using the described WHO criteria (Fig. 1), with an average decrease in the largest lesion size from $29.5 \pm 18 \mathrm{~mm}$ (mean \pm S.D., range: $13-60 \mathrm{~mm}$ ) to $19.5 \pm 13 \mathrm{~mm}$ (mean \pm S.D., range: $7-32 \mathrm{~mm}$ ). The tumor response to TACE was unrelated to the number and the size of liver metastases or to the decrease in the levels of tumor markers. One patient (patient number 1) who experienced a partial response after the first TACE cycle for a period of two years, underwent another TACE course due to disease progression, and disease stabilization was achieved. The overall time to tumor progression since the last TACE was 38 months (range: 8-126). 
Three out of the seven included patients were still

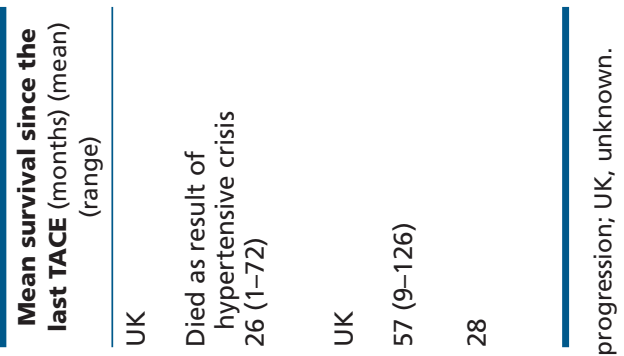

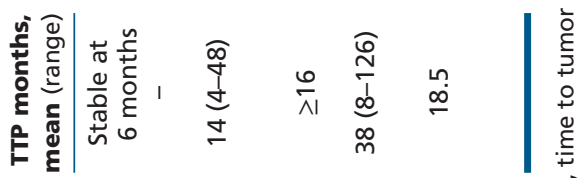
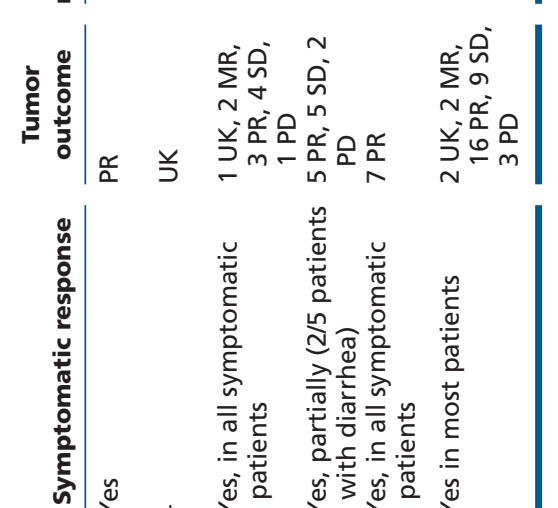

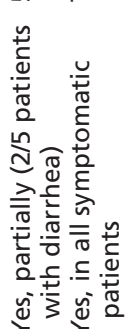

N
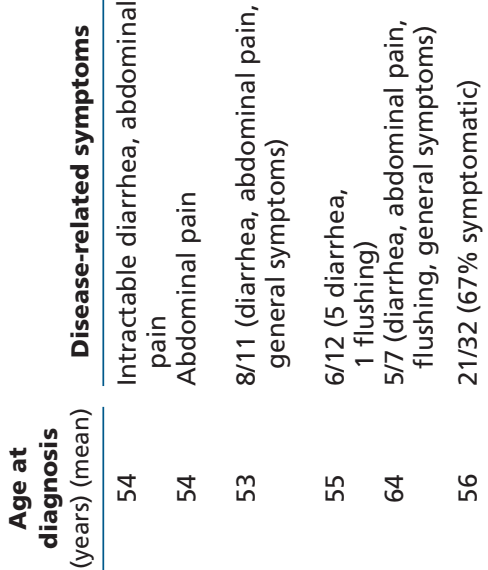

뉴 రु เ゚
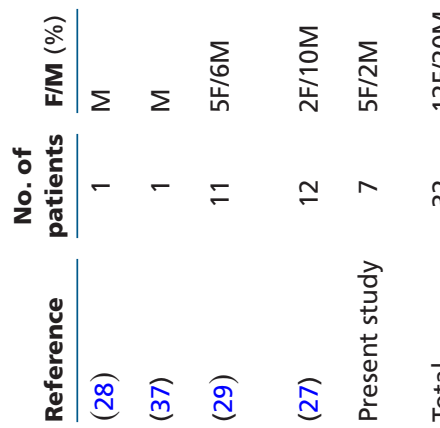
alive at the end of the follow-up period, whereas four patients died from progressive disease (an overall survival rate of $4.77 \pm 3.62$ years).

\section{Discussion}

The liver is a frequent site for metastatic medullary thyroid carcinoma, which is often disseminated throughout both hepatic lobes. Surgical resection or radiofrequency ablation (RFA) is considered in patients with isolated hepatic metastases, whereas chemotherapy is usually inefficient and rarely administered in patients with disseminated metastases $(22,35,36)$.

Data regarding trans-arterial liver embolization in the rare patients with MTC and progressive liver metastases are scarce and limited to case reports and small series (Table 3). In 1999, Isozaki et al. (28) reported for the first time the successful use of trans-catheter arterial embolization followed by percutaneous ethanol injection to liver metastases in a 54-year-old man with metastatic MTC, with intractable diarrhea and abdominal pain. The post-treatment CT scan demonstrated that the hepatic metastases were no longer enhancing, and on hepatic arteriogram, tumor stains indicating the presence of large tumors had disappeared. After six months of follow-up, there have been no signs of tumor regrowth or worsening of diarrhea.

In a study of liver chemoembolization in 11 patients with metastatic MTC, symptomatic response was noted in all patients, with biochemical and radiological responses in only 6 of them (55\%) (29). In another study of 12 patients with metastatic MTC (27), a partial radiological response occurred in $42 \%$, disease stabilization occurred in $42 \%$, with a duration of partial response and stabilization after a single TACE cycle exceeding 16 months. The extent of liver involvement was the main factor that influenced results, with best response in patients with less than $30 \%$ of the liver involved and with metastases size less than 30-mm in diameter. When disease progression occurred, patients could be offered repeated treatment, which often resulted in another partial tumor response, usually of shorter duration (27).

Our present series confirm the previous data, emphasizing that TACE appears to be an extremely effective therapeutic option for MTC patients with liver metastases, inducing both symptomatic and antiproliferative remission for prolonged lengths of time, and with a favorable adverse events profile (Table 3). 
Noteworthy, in our patient group, we have observed a beneficial effect in both smaller but also larger than $30 \mathrm{~mm}$ lesions and also in patients with liver metastatic involvement of up to $50 \%$. Moreover, the median time to tumor progression since the last TACE reached about 3 years, whereas symptomatic relief was reported in all symptomatic patients, with significant improvement in both specific (diarrhea, flushing and abdominal pain) as well as non-specific (weakness and weight loss) tumorrelated symptoms. These results are indeed important, as liver involvement represents a major reason for disease-related morbidity and mortality in patients with metastatic MTC.

In summary, metastatic medullary thyroid carcinoma is a rare condition; although a major limitation of the present study is the small number of patients included, our data are important as they highlight that the disease usually responds well to treatment with TACE, within a reasonable safety profile. It appears that TACE is usually well tolerated, inducing both clinical improvement and tumor response for prolonged periods of time in the majority of patients with MTC-related liver metastases, improving the quality of life of these patients and postponing the use of systemic and more complex therapies, such as tyrosine kinase inhibitors. Before chemoembolization, patients should be carefully evaluated in terms of adequate renal and hepatic function, and the presence of possible pheochromocytomas in patients with MEN2 syndromes should be ruled out (1). Most of the MTC patients with liver involvement will also have metastases to lymph nodes, bones and other organs, and therefore, will require systemic therapies (e.g., tyrosine kinases inhibitors, bisphosphonates, etc.) when multiple tumor-sites progression occurs. However, TACE should always be considered early in the treatment of these patients; as the deterioration in liver function is a major factor determining prognosis, the appropriate candidates for TACE should be the patients in whom the disease progression occurs mainly in the liver, and irrespective of the presence of extrahepatic metastases.

Prospective multicenter randomized studies, including larger number of MTC patients with liver metastases, would be optimal for definition of the best candidates for TACE, for treatment timing and for evaluating its efficacy in terms of time to tumor progression and longterm survival. However, due to the extreme rarity of this condition, the probability for such trials is remote, and therefore, clinicians who manage these patients will most probably have to rely on personal experience and data from retrospective studies, such as ours.
Declaration of interest

The authors declare that there is no conflict of interest that could be perceived as prejudicing the impartiality of the research reported.

\section{Funding}

This research did not receive any specific grant from any funding agency in the public, commercial or not-for-profit sector.

\section{References}

1 Wells SA Jr, Asa SL, Dralle H, Elisei R, Evans DB, Gagel RF, Lee N, Machens A, Moley JF, Pacini F et al. Revised American Thyroid Association guidelines for the management of medullary thyroid carcinoma. Thyroid 201525 567-610. (doi:10.1089/thy.2014.0335)

2 Kloos RT, Eng C, Evans DB, Francis GL, Gagel RF, Gharib H, Moley JF, Pacini F, Ringel MD, Schlumberger $\mathrm{M}$ et al. Medullary thyroid cancer: management guidelines of the American Thyroid Association. Thyroid 200919 565-612. (doi:10.1089/thy.2008.0403)

3 Elisei R, Schlumberger M, Driedger A, Reiners C, Kloos RT, Sherman SI, Haugen B, Corone C, Molinaro E, Grasso L et al. Follow-up of low-risk differentiated thyroid cancer patients who underwent radioiodine ablation of postsurgical thyroid remnants after either recombinant human thyrotropin or thyroid hormone withdrawal. Journal of Clinical Endocrinology and Metabolism 200994 4171-4179. (doi:10.1210/jc.2009-0869)

4 Chen H, Sippel RS, O'Dorisio MS, Vinik AI, Lloyd RV \& Pacak K. The North American Neuroendocrine Tumor Society consensus guideline for the diagnosis and management of neuroendocrine tumors: pheochromocytoma, paraganglioma, and medullary thyroid cancer. Pancreas 201039 775-783. (doi:10.1097/MPA.0b013e3181ebb4f0)

5 Brandi ML, Gagel RF, Angeli A, Bilezikian JP, Beck-Peccoz P, Bordi C, Conte-Devolx B, Falchetti A, Gheri RG, Libroia A et al. Guidelines for diagnosis and therapy of MEN type 1 and type 2. Journal of Clinical Endocrinology and Metabolism 200186 5658-5671. (doi:10.1210/ jcem.86.12.80700)

6 Grozinsky-Glasberg S, Benbassat CA, Tsvetov G, Feinmesser R, Peretz H, Shimon I \& Lapidot M. Medullary thyroid cancer: a retrospective analysis of a cohort treated at a single tertiary care center between 1970 and 2005. Thyroid 200717 549-556. (doi:10.1089/thy.2006.0229)

7 Leboulleux S, Baudin E, Travagli JP \& Schlumberger M. Medullary thyroid carcinoma. Clinical Endocrinology 200461 299-310. (doi:10.1111/j.1365-2265.2004.02037.x)

8 Mirallie E, Vuillez JP, Bardet S, Frampas E, Dupas B, Ferrer L, FaivreChauvet A, Murat A, Charbonnel B, Barbet J et al. High frequency of bone/bone marrow involvement in advanced medullary thyroid cancer. Journal of Clinical Endocrinology and Metabolism 200590 779-788. (doi:10.1210/jc.2004-1500)

9 Brierley J, Tsang R, Simpson WJ, Gospodarowicz M, Sutcliffe S \& Panzarella T. Medullary thyroid cancer: analyses of survival and prognostic factors and the role of radiation therapy in local control. Thyroid 19966 305-310. (doi:10.1089/thy.1996.6.305)

10 Hyer SL, Vini L, A'Hern R \& Harmer C. Medullary thyroid cancer: multivariate analysis of prognostic factors influencing survival. European Journal of Surgical Oncology 26 686-690. (doi:10.1053/ ejso.2000.0981)

11 Abe K, Adachi I, Miyakawa S, Tanaka M, Yamaguchi K, Tanaka N, Kameya T \& Shimosato Y. Production of calcitonin, adrenocorticotropic hormone, and beta-melanocyte-stimulating hormone in tumors derived from amine precursor uptake and decarboxylation cells. Cancer Research 197737 4190-4194.

12 Ishikawa N \& Hamada S. Association of medullary carcinoma of the thyroid with carcinoembryonic antigen. British Journal of Cancer 1976 34 111-115. (doi:10.1038/bjc.1976.133) 
13 Mato E, Matias-Guiu X, Chico A, Webb SM, Cabezas R, Berna L \& De Leiva A. Somatostatin and somatostatin receptor subtype gene expression in medullary thyroid carcinoma. Journal of Clinical Endocrinology and Metabolism 199883 2417-2420. (doi:10.1210/ jcem.83.7.4955)

14 Miyauchi A, Matsuzuka F, Hirai K, Yokozawa T, Kobayashi K, Ito Y, Nakano K, Kuma K, Futami H \& Yamaguchi K. Prospective trial of unilateral surgery for nonhereditary medullary thyroid carcinoma in patients without germline RET mutations. World Journal of Surgery 200226 1023-1028. (doi:10.1007/s00268-002-6665-1)

15 Weber T, Schilling T, Frank-Raue K, Colombo-Benkmann M, Hinz U, Ziegler R \& Klar E. Impact of modified radical neck dissection on biochemical cure in medullary thyroid carcinomas. Surgery 2001130 1044-1049. (doi:10.1067/msy.2001.118380a)

16 Machens A, Holzhausen HJ \& Dralle H. Contralateral cervical and mediastinal lymph node metastasis in medullary thyroid cancer: systemic disease? Surgery 2006139 28-32. (doi:10.1016/j. surg.2005.06.018)

17 Kapiteijn E, Schneider TC, Morreau H, Gelderblom H, Nortier JW $\&$ Smit JW. New treatment modalities in advanced thyroid cancer. Annals of Oncology 201223 10-18. (doi:10.1093/annonc/mdr117)

18 Pelizzo MR, Boschin IM, Bernante P, Toniato A, Piotto A, Pagetta C, Nibale O, Rampin L, Muzzio PC \& Rubello D. Natural history, diagnosis, treatment and outcome of medullary thyroid cancer: 37 years experience on 157 patients. European Journal of Surgical Oncology 200733 493-497. (doi:10.1016/j.ejso.2006.10.021)

19 Wells SA Jr, Robinson BG, Gagel RF, Dralle H, Fagin JA, Santoro M, Baudin E, Elisei R, Jarzab B, Vasselli JR et al. Vandetanib in patients with locally advanced or metastatic medullary thyroid cancer: a randomized, double-blind phase III trial. Journal of Clinical Oncology 201230 134-141. (doi:10.1200/JCO.2011.35.5040)

20 Kurzrock R, Sherman SI, Ball DW, Forastiere AA, Cohen RB, Mehra R, Pfister DG, Cohen EE, Janisch L, Nauling F et al. Activity of XL184 (Cabozantinib), an oral tyrosine kinase inhibitor, in patients with medullary thyroid cancer. Journal of Clinical Oncology 201129 2660-2666. (doi:10.1200/JCO.2010.32.4145)

21 Lam ET, Ringel MD, Kloos RT, Prior TW, Knopp MV, Liang J, Sammet S, Hall NC, Wakely PE Jr, Vasko VV et al. Phase II clinical trial of sorafenib in metastatic medullary thyroid cancer. Journal of Clinical Oncology 201028 2323-2330. (doi:10.1200/JCO.2009.25.0068)

22 Orlandi F, Caraci P, Berruti A, Puligheddu B, Pivano G, Dogliotti L \& Angeli A. Chemotherapy with dacarbazine and 5-fluorouracil in advanced medullary thyroid cancer. Annals of Oncology 19945 763-765.

23 Petursson SR. Metastatic medullary thyroid carcinoma. Complete response to combination chemotherapy with dacarbazine and 5-fluorouracil. Cancer 198862 1899-1903. (doi:10.1002/10970142(19881101)62:9<1899::AID-CNCR2820620905>3.0.CO;2-C)

24 Nocera M, Baudin E, Pellegriti G, Cailleux AF, Mechelany-Corone C \& Schlumberger M. Treatment of advanced medullary thyroid cancer with an alternating combination of doxorubicin-streptozocin and 5 FU-dacarbazine. British Joutnal of Cancer 200083 715-718. (doi:10.1054/bjoc.2000.1314)
25 Salavati A, Puranik A, Kulkarni HR, Budiawan H \& Baum RP. Peptide receptor radionuclide therapy (PRRT) of medullary and nonmedullary thyroid cancer using radiolabeled somatostatin analogues. Seminars in Nuclear Medicine 201646 215-224. (doi:10.1053/j.semnuclmed.2016)

26 Behr TM \& Behe MP. Cholecystokinin-B/Gastrin receptor-targeting peptides for staging and therapy of medullary thyroid cancer and other cholecystokinin-B receptor-expressing malignancies. Biopolymers 200266 399-418. (doi:10.1002/bip.10356)

27 Fromigue J, De Baere T, Baudin E, Dromain C, Leboulleux S \& Schlumberger M. Chemoembolization for liver metastases from medullary thyroid carcinoma. Journal of Clinical Endocrinology and Metabolism 200691 2496-2499. (doi:10.1210/jc.2005-2401)

28 Isozaki T, Kiba T, Numata K, Saito S, Shimamura T, Kitamura T, Morita K, Tanaka K \& Sekihara H. Medullary thyroid carcinoma with multiple hepatic metastases: treatment with transcatheter arterial embolization and percutaneous ethanol injection. Internal Medicine 199938 17-21. (doi:10.2169/internalmedicine.38.17)

29 Lorenz K, Brauckhoff M, Behrmann C, Sekulla C, Ukkat J, Brauckhoff K, Gimm O \& Dralle H. Selective arterial chemoembolization for hepatic metastases from medullary thyroid carcinoma. Surgery 2005138 986-993. (doi:10.1016/j.surg.2005.09.020)

30 Rammohan A, Sathyanesan J, Ramaswami S, Lakshmanan A, SenthilKumar P, Srinivasan UP, Ramasamy R \& Ravichandran P. Embolization of liver tumors: past, present and future. World Journal of Radiology 20124 405-412. (doi:10.4329/wjr.v4.i9.405)

31 Chung JW, Park JH, Han JK, Choi BI, Han MC, Lee HS \& Kim CY. Hepatic tumors: predisposing factors for complications of transcatheter oily chemoembolization. Radiology 1996198 33-40. (doi:10.1148/radiology.198.1.8539401)

32 Yinglu F, Changquan L, Xiaofeng Z, Bai L, Dezeng Z \& Zhe C. A new way: alleviating postembolization syndrome following transcatheter arterial chemoembolization. Journal of Alternative and Complementary Medicine 200915 175-181. (doi:10.1089/acm.2008.0093)

33 Moon E, Tam MD, Kikano RN \& Karuppasamy K. Prophylactic antibiotic guidelines in modern interventional radiology practice. Seminars in Interventional Radiology 201027 327-337. (doi:10.105 5/s-0030-1267853)

34 Rindi G, de Herder WW, O’Toole D \& Wiedenmann B. Consensus guidelines for the management of patients with digestive neuroendocrine tumors: the second event and some final considerations. Neuroendocrinology 200887 5-7. (doi:10.1159/000109975)

35 Frilling A, Weber F, Tecklenborg C \& Broelsch CE. Prophylactic thyroidectomy in multiple endocrine neoplasia: the impact of molecular mechanisms of RET proto-oncogene. Langenbeck's Archives of Surgery 2003388 17-26. (doi:10.1007/s00423-003-0368-4)

36 Wertenbroek MW, Links TP, Prins TR, Plukker JT, van der Jagt EJ \& de Jong KP. Radiofrequency ablation of hepatic metastases from thyroid carcinoma. Thyroid 200818 1105-1110. (doi:10.1089/thy.2008.0080)

37 Machens A, Behrmann C \& Dralle H. Chemoembolization of liver metastases from medullary thyroid carcinoma. Annals of Internal Medicine 2000132 596-597. (doi:10.7326/0003-4819-132-7200004040-00028)

Received 23 November 2016

Revised version received 12 January 2017

Accepted 18 January 2017 